\title{
Impact of the COVID-19 Pandemic on Surgical Oncology in Europe: Results of a European Survey
}

\author{
Christian Stöss $^{\text {a }}$ Marcella Steffani $^{a} \quad$ Ilaria Pergolini $^{a}$ Daniel Hartmann ${ }^{a}$ \\ Dejan Radenkovic $^{b} \quad$ Alexander Novotny $^{a}$ Helmut Friess $^{a}$ Michael W. Müller ${ }^{a, c}$ \\ aDepartment of Surgery, School of Medicine, Klinikum rechts der Isar, Technical University of Munich, \\ Munich, Germany; ${ }^{b}$ Clinic of Digestive Surgery, Clinical Centre of Serbia, Faculty of Medicine, University of \\ Belgrade, Belgrade, Serbia; 'Clinical Centre Ludwigsburg-Bietigheim, Clinic of General and Visceral Surgery, \\ Ludwigsburg-Bietigheim, Germany
}

\section{Keywords}

COVID-19 pandemic · Surgical care · Surgical oncology · European surgery

\begin{abstract}
Background: The first COVID-19 pandemic wave hit most of the health-care systems worldwide. The present survey aimed to provide a European overview on the COVID-19 impact on surgical oncology. Methods: This anonymous online survey was accessible from April 24 to May 11, 2020, for surgeons ( $n=298$ ) who were contacted by the surgical society European Digestive Surgery. The survey was completed by 88 surgeons (29.2\%) from 69 different departments. The responses per department were evaluated. Results: Of the departments, $88.4 \%(n=61 / 69)$ reported a lower volume of patients in the outpatient clinic; $69.1 \%(n=47 / 68)$ and $75.0 \%$ $(n=51 / 68)$ reported a reduction in hospital bed and the operating room capacity, respectively. As a result, the participants reported an average reduction of $29.3 \%$ for all types of oncological resections surveyed in this questionnaire. The strongest reduction was observed for oncological resections of hepato-pancreatico-biliary (HPB) cancers. Of the inter-
\end{abstract}

viewed surgeons, $68.7 \%(n=46 / 67)$ agreed that survival outcomes will be negatively impacted by the pandemic. Conclusion: The first COVID-19 pandemic wave had a significant impact on surgical oncology in Europe. The surveyed surgeons expect an increase in the number of unresectable cancers as well as poorer survival outcomes due to cancellations of follow-ups and postponements of surgeries.

(C) 2021 S. Karger AG, Basel

\section{Introduction}

The first COVID-19 pandemic wave has resulted in a significant restructuring of health care systems and societies in order to prevent high infection and mortality rates. However, the current number of infections show that the crisis is ongoing and far from being under control [1]. For oncological surgeons, the circumstances caused by the pandemic pose a dichotomous problem: firstly, surgeons must be prepared to offer acute (oncological) surgery for COVID-19 positive patients, and secondly, they must maintain high standards of surgical care for cancer patients. During the first wave, elective surgical 
Table 1. General characteristics

\begin{tabular}{|c|c|}
\hline Variable & $n(\%)$ \\
\hline \multicolumn{2}{|l|}{ Surgeons } \\
\hline \multicolumn{2}{|l|}{ EDS member } \\
\hline Yes & $69(81.2)$ \\
\hline No & $15(17.6)$ \\
\hline Unknown & $1(1.2)$ \\
\hline \multicolumn{2}{|l|}{ Sex } \\
\hline Male & $74(87.1)$ \\
\hline Female & $11(12.9)$ \\
\hline \multicolumn{2}{|l|}{ Age } \\
\hline$<35$ & $31(36.5)$ \\
\hline $35-54$ & $37(43.5)$ \\
\hline$\geq 55$ & $17(20.0)$ \\
\hline \multicolumn{2}{|l|}{ Profession ${ }^{1}$} \\
\hline Head of department & $25(29.4)$ \\
\hline Consultant & $24(28.2)$ \\
\hline Board certified surgeon & $22(25.9)$ \\
\hline Resident & $14(16.5)$ \\
\hline \multicolumn{2}{|l|}{ Surgical specialty $^{2}$} \\
\hline $\mathrm{HPB}$ & $50(32.3)$ \\
\hline Colorectal & $48(31.0)$ \\
\hline Upper GI & $37(23.9)$ \\
\hline Endocrine & $9(5.8)$ \\
\hline Liver transplant & $2(1.3)$ \\
\hline Vascular & $1(0.6)$ \\
\hline Gynaecology and obstetrics & $2(1.3)$ \\
\hline Thoracic & $1(0.6)$ \\
\hline Hernia & $1(0.6)$ \\
\hline Unspecified & $4(2.6)$ \\
\hline \multicolumn{2}{|l|}{ Departments } \\
\hline \multicolumn{2}{|l|}{ Type of hospital } \\
\hline University hospital & $34(49.3)$ \\
\hline General hospital (university-affiliated) & $16(23.2)$ \\
\hline General hospital (non-university) & $14(20.3)$ \\
\hline Private practice & $3(4.3)$ \\
\hline Unspecified & $2(2.9)$ \\
\hline \multicolumn{2}{|l|}{ Size of hospital (number of beds) } \\
\hline$<100$ & $1(1.4)$ \\
\hline $100-200$ & $5(7.2)$ \\
\hline $201-500$ & $24(34.8)$ \\
\hline $501-1,000$ & $24(34.8)$ \\
\hline$>1,000$ & $15(21.7)$ \\
\hline \multicolumn{2}{|c|}{ Intensive care capacity (number of intensive care beds) } \\
\hline$<10$ & $6(9.0)$ \\
\hline $10-20$ & $26(38.8)$ \\
\hline $21-50$ & $25(37.3)$ \\
\hline $51-100$ & $9(13.4)$ \\
\hline$>100$ & $1(1.5)$ \\
\hline
\end{tabular}

HPB, hepato-pancreatico-biliary; GI Gastrointestinal; EDS, European Digestive Surgery. ${ }^{1}$ Obligatory to fill in. ${ }^{2}$ Multiple answers were possible. procedures were postponed to provide intensive care beds, respiratory equipment, and personnel to treat COVID-19 patients. To this end, instructions for triage and urgent surgical interventions have been published by national surgical societies and associations [2-4]. The pandemic's impact on oncological patients is not foreseeable, but first results indicate a negative influence of $\mathrm{CO}$ VID-19 on the oncological outcome due to delays in diagnostics [5]. Political interventions are therefore needed to prevent both a further increase in cancer-related mortality and COVID-19-related deaths in cancer patients. In this regard, health-care systems and centres must implement concrete strategies to continue oncological treatment. It is important that these strategies incorporate surgeon's experience.

The present survey aims to investigate the impact of the first COVID-19 pandemic wave on surgical oncology by collecting data on surgeons' experiences regarding the reduction of beds as well as the operating room capacity with special respect to the reduction of cancer resections. The results may be of interest for physicians, health-care providers, and politicians of countries, who are still fighting against high infection rates or awaiting the peak of the next COVID-19 pandemic wave.

\section{Methods}

\section{Study Design}

For the present cross-sectional study, with the support of the United European Gastroenterology-affiliated society European Digestive Surgery (EDS), a European association of surgeons, 298 surgeons in Europe were invited to an anonymous online survey from April 24 to May 11, 2020, via email. Participation in the survey was voluntary and no personal data for analysis were collected. Surgeons who took part in the survey and wished to be named as a collaborator were listed in online suppl. Table 1 (for all online suppl. material, see www.karger.com/doi/10.1159/000515186). The survey was completed by 88 surgeons contacted (29.5\%). Three non-European responses were excluded from analysis and multiple answers from one department were combined (median was calculated when appropriate). Thus, 69 department answers were analysed.

\section{Survey}

A commercial provider was used for the online survey (Google Forms, https://docs.google.com/forms; Google Inc., Mountain View, CA, USA). The survey consists of 64 questions and statements divided into 7 categories. A combination of a bipolar Likert scale ("Strongly agree," "Agree," "Neutral," "Disagree," and "Strongly disagree"), close-ended (yes/no/unknown), and openended questions was applied. Only one answer was possible if it was not otherwise indicated.

In the first section, baseline characteristics (10 questions) were collected from the respondents. The second (COVID-19 informa- 


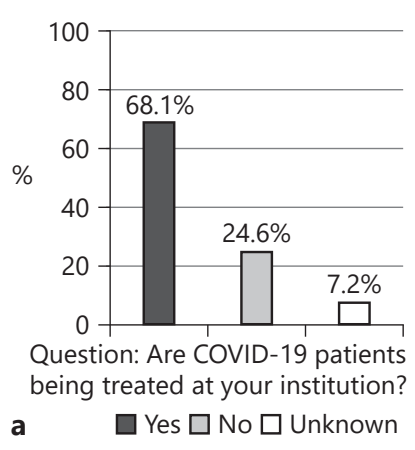

$\%$

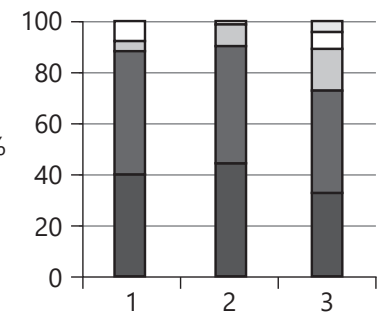

1 The number of oncological patients in the outpatient clinic has decreased. 2 In fear of a COVID-19 infection, patients cancel their outpatient appointments. 3 The appointment for planning an oncological resection is postponed if a patient is tested positive for COVID-19.

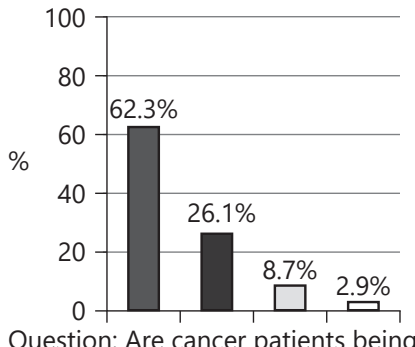

Question: Are cancer patients being screened for COVID-19 prior to tumour surgery?

$\square$ Yes, all cancer patients will be screened prior to surgery.

Yes, but only selected patients (e.g., age, co-morbidities, etc.).

$\square$ No, there is no screening at all. b $\square$ Unspecified

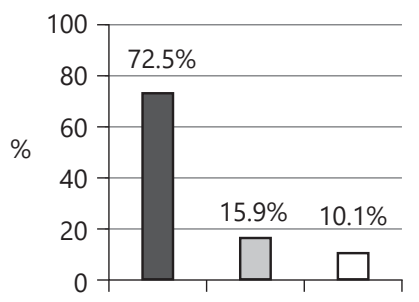

Statement: Patients wish to postpone their operation appointments due to COVID-19.

e $\square$ Yes $\square$ No $\square$ Unknown

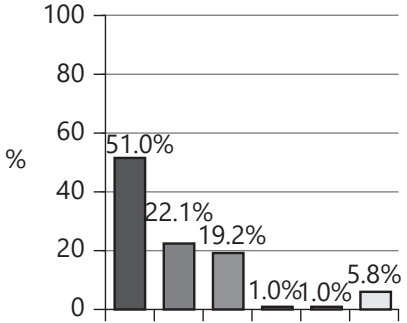

Please indicate the diagnostic tools that are used for COVID-19 screening.

\section{$\square$ PCR $\square$ Chest X-ray}

$\square$ CT chest scan $\quad \square$ Unspecified

$\square$ Serology (antibody test)

c $\square$ Questionnaire

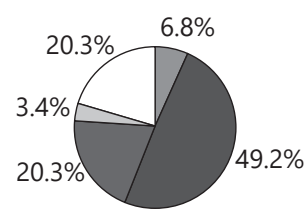

Estimated delay due to postponement of the operation (see Figure 1e).

$\square$ Less than 2 weeks $\square$ 2-4 weeks

4-8 weeks $\square$ More than 8 weeks f $\square$ Currently not foreseeable

Strongly agree $\square$ Agree $\square$ Neutral $\square$ Disagree

d $\square$ Strongly disagree

Fig. 1. Graphical depiction of the results of question section No. 2 (COVID-19 information, a-c) and No. 3 (outpatient clinics, $\mathbf{d - f}$ ). a Number of departments currently treating COVID-19 patients. b COVID-19 screening at the participating centres. c Diagnostic screening tools for COVID-19. d Effects on outpatient clinics. e Results of whether patients would like to reschedule their surgery appointments. $\mathbf{f}$ Results on the extent to which patients postpone appointments.

tion, 9 questions) and third section (outpatient clinics, 5 questions) contained questions about the impact of the pandemic on outpatient clinics. The fourth section (tumour diagnostics, 7 questions) sought availability of various diagnostic and staging tools, the fifth category (oncological treatment, 23 questions) requested the number of resections for different types of cancer before the COVID-19 crisis and the relative reduction, the sixth category contained questions regarding tumour follow-up (4 questions), and the seventh category consisted of 5 questions regarding future aspects. The complete survey can be found in online suppl. data file 1 .

\section{Statistical Analysis}

Descriptive data analysis was performed with Microsoft Excel 2019 (Microsoft, Redmond, WA, USA). Categorial variables are given as absolute and relative frequencies. Continuous variables are presented as mean value with single standard deviation. Numbers of cancer resections are calculated as median and interquartile range.

\section{Results}

\section{General Characteristics}

Overall, 81.2\% ( $n=69 / 85)$ of all interviewed surgeons were active members of EDS. Sixty-nine departments from 24 different European countries are represented in this 


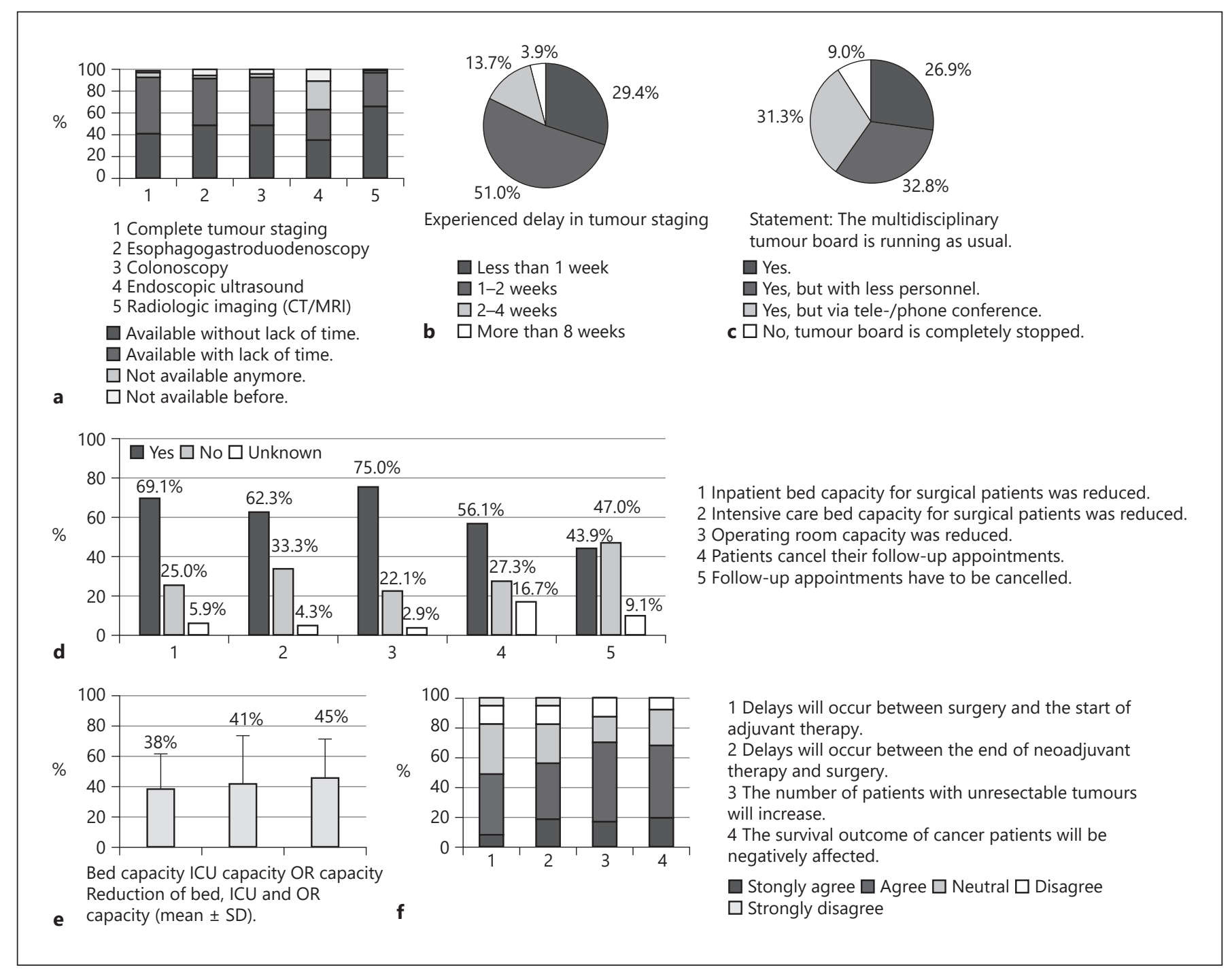

Fig. 2. Graphical depiction of the results of question section No. 4 (tumour diagnostics, a-c) and No. 5 (oncological treatment, $\mathbf{d - f}$ ) of the survey. a Impact on various tumour diagnosis units. $\mathbf{b}$ Presentation of the extent of the perceived delay in diagnosis. $\mathbf{c}$ Report on the continuation of the tumour board. $\mathbf{d}$ Effects on capacities of surgical departments and oncological follow-ups. e Mean reduction $( \pm \mathrm{SD})$ of operating room, bed, and ICU bed capacity. $\mathbf{f}$ Expected effects on treatment plans and cancer outcome. SD, standard deviation.

survey (online suppl. Fig. 1). Most departments are located in Ukraine $(n=13 / 69,19 \%)$, Romania $(n=5 / 69,7 \%)$, Italy $(n=5 / 69,7 \%)$, and Germany $(n=5 / 69,7 \%)$. See Table 1 for complete general characteristics of the study cohort.

\section{COVID-19 Pandemic and Virus Screening}

COVID-19 patients were treated by $68.1 \%(n=47 / 69)$ of the departments (Fig. 1a). Cancer patients treated at these institutions were screened in $62.3 \%(n=43 / 69)$ for COVID-19 infection (Fig. 1b). Of all the departments, $26.1 \%(n=18 / 69)$ screened selected patients only (e.g., age and comorbidities), and $8.7 \%(n=6 / 69)$ did not screen the patients prior to treatment. Multiple tools were used to test for COVID-19 infection (Fig. 1c). Most commonly (51.0\%; $n=53 / 104)$ PCR was applied followed by CT chest scan $(22.1 \% ; n=23 / 104)$ and serology diagnostics $(19.2 \% ; n=20 / 104)$.

\section{Outpatient Clinics}

Of all departments, $88.4 \%(n=61 / 69)$ agreed entirely or agreed with the statement that the number of oncological patients decreased during consultation hours 
Table 2. Effect on the number of oncological resections

\begin{tabular}{lcccc}
\hline Cancer type & $n$ & $\begin{array}{l}\text { Annual number of resections } \\
\text { before COVID-19 (average } \\
\text { of last 3 years), median (IQR) }\end{array}$ & $n \quad \begin{array}{l}\text { Reduction of } \\
\text { resections (in \%), } \\
\text { mean }( \pm \text { SD) }\end{array}$ \\
\hline Pancreatic cancer & 64 & $28(10-50)$ & 66 & $31.6( \pm 35.4)$ \\
Oesophageal cancer & 59 & $8(0-20)$ & 60 & $19.0( \pm 32.1)$ \\
Gastric cancer & 58 & $30(20-50)$ & 60 & $27.4( \pm 33.5)$ \\
Colorectal cancer & 59 & $110(60-205)$ & 61 & $30.0( \pm 30.3)$ \\
Hepatic cancer and hepatic metastasis & 57 & $20(5-50)$ & 60 & $32.8( \pm 36.4)$ \\
Bile duct cancer & 61 & $10(5-15)$ & 62 & $33.8( \pm 39.5)$ \\
Neuroendocrine tumours & 57 & $10(5-20)$ & 61 & $30.2( \pm 39.4)$
\end{tabular}

$\mathrm{IQR}$, interquartile range; $\mathrm{SD}$, standard deviation; $n$, number of answers given.

(Fig. 1d). The statement that patients cancelled their appointment due to the fear of COVID-19 infection was supported or fully supported in $89.9 \%(n=62 / 69)$. If patients tested positive, operations were mostly postponed (72.5\%; $n=50 / 69$ totally agreed or agreed to this statement). The statement "Patients wish to postpone their operation appointments due to COVID-19." (Fig. 1e) was answered positively in $72.5 \%(n=50 / 69)$ and negatively in $15.9 \%(n=11 / 69)$. The participating departments were asked about the expected delay due to postponing appointments and operations. Most departments (49.2\%; $n$ $=29 / 59)$ expected a delay of $2-4$ weeks and $20.3 \%(n=$ 12/59) reported a delay of up to $4-8$ weeks (Fig. 1f).

\section{Tumour Diagnostics}

In $92.8 \%$ of all centres that answered the question $(n=$ 64/69), diagnostics for complete tumour staging was available. Most diagnostic procedures continued to perform without lack of time or with a delay of 1-2 weeks. Endoscopy departments were more affected than diagnostics units. The continuation of interdisciplinary tumour boards is essential for the quality of care in oncological patients. Of all departments, 9.0\% $(n=6 / 67)$ reported a complete stop of tumour boards (Fig. 2a-c).

\section{Effects on Surgical Oncology}

Restructuring of health-care institutions were initiated to prepare for the treatment of COVID-19 patients. In the present survey, 69.1\% $(n=47 / 68)$ departments reported a decrease in inpatient bed capacities of $38.4 \pm 23.0 \%$. Of the centres, $75.0 \%(n=51 / 68)$ indicated a mean reduction in the operating room capacity of $45.5 \pm 25.1 \%$ (Fig. $2 \mathrm{~d}$, e). In addition, the majority $(56.1 \% ; n=37 / 66)$ experienced that patients cancelled their follow-up appointments. The number of different tumour resections per year in these centres and the extent of reduced resections due to the COVID-19 pandemic is shown in Table 2. The mean reduction for all cancer resections was calculated at 29.3\%. Resections for hepato-pancreatico-biliary (HPB) cancers were the most affected (31.6-33.8\%). Further, $49.2 \%(n=32 / 65)$ entirely or partially agreed with the statement that the start of adjuvant chemotherapy will be delayed (Fig. 2f). In addition, $56.1 \%(n=27 / 66)$ partially or fully agreed that the time interval between neoadjuvant treatment and surgery will increase, and 68.7\% $(n=46 / 67)$ partially or agreed in entirety that the survival outcome of tumour patients could be at risk. Finally, the department representatives answered questions regarding future aspects of the COVID-19 pandemic, which are summarized in online suppl. Figure 2.

\section{Discussion}

The results of the present survey indicate a considerable influence of COVID-19-related restructuring, such as reductions in inpatient bed and operating room capacity, on surgical departments. In general, our results show that there is an urgent need to prepare for an increase in the number of irresectable cancers as well as for more complex resections due to delays from the pandemic. Furthermore, the participants expect a negative impact on the survival outcome of cancer patients. This expectation is supported by recent publications showing that cancer outcome will be negatively affected by the COVID-19 pandemic [6-8]. However, evidence also exists showing that strict implementation of separation and screening protocols can lead to safe surgeries to avoid an increase of mortality related to COVID-19 [9]. Furthermore, other cancer treatments such as chemotherapy or immunother- 
apy do not increase the risk of death from COVID-19 [10]. Therefore, strategies and guidelines must now be introduced that take into account the clinical and practical experience of surgeons who are confronted with the abovementioned problems on a daily basis in order to prevent further delays in the treatment of cancer patients.

Our survey confirms that surgeons have experienced patient cancellation of appointments for planned surgery or diagnostic follow-ups for fear of COVID-19 infection. Overall, a reduction in the number of consultations was observed. As follow-up appointments are essential in oncology, telemedicine applications could bridge the gap until large-scale vaccination programmes are implemented to enable consultations without the risk of infection. As telemedicine is well accepted, this might be a possible solution for the outpatient care of oncological patients [11].

Since several gastroenterology societies have recommended suspension of elective endoscopic procedures, an effect on tumour diagnostics was expected [12, 13]. However, the results of this survey show that diagnostics required for complete tumour staging were minimally affected with an expected pandemic-related delay of 1-2 weeks. Further, the continuity of interdisciplinary tumour boards is crucial for the quality of care in oncological treatment plans $[14,15]$. However, in $9.0 \%$ of all cases, the tumour board was completely discontinued. It is important to maintain tumour diagnostics in general without any delay. For this reason, the establishment of diagnostic units that are kept as free as possible from COVID-19-positive patients (e.g., by spatial separation or extensive screening of patients) is suggested as an essential measure.

The focus of this study was to provide initial estimates and reference points for future research on the impact of the COVID-19 pandemic on oncological resection rates. As expected, the participating surgical departments perceive a reduction in tumour resections of all types. On average, the number of resections (for all questioned types of cancer) was reduced by almost one-third, which is consistent with a recent publication that estimates $38 \%$ of all cancer surgeries in all disciplines worldwide were cancelled due to the COVID-19 crisis [16]. In a previous survey by the European-African HPB Association, which examined the effect of the COVID-19 pandemic on the management of HPB tumours, most participants reported a reduction of operation room activity between 26 and $50 \%$ [17]. This result is comparable to our findings, which show that resections for HPB cancers were the most affected. All cancelled operations must be performed in near future in addition to the elective surgery schedule. In this context, the number of inoperable cancers may in- crease due to delays in diagnostics and surgery. The need for strategic rescheduling of operations is urgent. Exemplary strategic approaches have been published recently $[18,19]$. Another approach focuses on the selection and transformation of hospitals into infection-free cancer centres ("cancer hubs") to enable the safe treatment of patients separated from COVID-19 patients [20, 21]. Health care facilities for cancer care can be identified on the basis of performance criteria (e.g., extent of specialised cancer operations) and the regional care infrastructure. Overall, this should ensure continuity of care for essential cancer treatments while providing sufficient capacity for the medical care of COVID-19 patients.

The present survey is subject to limitations. The data collected are based on subjective assessments of the participants and therefore represent a potential source of bias. Furthermore, the participation rate in the survey was rather low (29.5\%), which might present a sampling bias. Another issue that needs to be addressed is the heterogeneity of the participant structure. The survey, which was conducted in different European countries that were affected differently by the COVID-19 pandemic in terms of severity and timing of the outbreak, may have been influenced by this. In addition, some highly populated European countries with a high number of cancer surgeries were under-represented in the survey. Furthermore, the results should only be considered as preliminary reference points for further studies on the effect of COVID-19 on the oncological treatment of our patients.

During the survey period, surgical departments faced an early stage of the COVID-19 pandemic and future effects are still difficult to predict and many uncertainties persist. However, it seems inevitable that the reduction in surgical capacity and the discontinuation of elective operations will compromise the survival outcome of oncological patients. Follow-up surveys and investigations must provide further evidence on this topic in the future. The coordination of European supranational and national measures for upholding care and treatment of oncological patients in the time of an ongoing pandemic must be reliably established. Our findings can inform adaptation of health care strategies to continue oncological surgery in the event of future pandemic waves.

\section{Acknowledgements}

The authors thank Dr. Henryk Haffer (Charité School of Medicine Berlin, Germany) and Dr. Sangeethabalasri Pugazhendhi (Stanford University School of Medicine, Stanford, CA, USA) for their support and scientific advice in conducting this study. 


\section{Statement of Ethics}

According to the Professional code of conduct for doctors in Bavaria $\$ 15$ (1), an Ethics Committee vote or support by the competent authority is not required for the presented investigation. Written informed consent has been obtained from all the participants included in this study.

\section{Conflict of Interest Statement}

Dres. Hartmann and Friess are editorial board members of $\mathrm{Di}$ gestive Surgery. The authors have no conflicts of interest to declare.

\section{Funding Sources}

Not applicable.

\section{Author Contributions}

All authors contributed to the study conception and design. Material preparation, data collection, and analysis were performed by Christian Stöss, Marcella Steffani, and Ilaria Pergolini. The first draft of the manuscript was written by Christian Stöss, Daniel Hartmann, Michael W. Müller, Dejan Radenkovic, Alexander Novotny, and Helmut Friess, and all authors commented on the previous versions of the manuscript. All authors read and approved the final manuscript.

\section{References}

1 Coronavirus Resource Center, Johns Hopkins University. 2020. Available from: https:// coronavirus.jhu.edu/map.html Accessed 2020 Aug 30.

2 DGAV, Rundschreiben zu OP-Indikationen. 2020. Available from: https://www.dgav.de/ dgav/aktuelles/aktuelles-aus-der-dgav/article/dgav-rundschreiben-zu-op-indikationen. html Accessed 2020 May 3.

3 Royal College of Surgeons of England. 2020. Available from: https://www.rcseng.ac.uk/ coronavirus/ Accessed 2020 May 24.

4 American College of Surgeons. 2020. Available from: https://www.facs.org/covid-19 Accessed 2020 May 24.

5 Maringe C, Spicer J, Morris M, Purushotham A, Nolte E, Sullivan R, et al. The impact of the COVID-19 pandemic on cancer deaths due to delays in diagnosis in England, UK: a national, population-based, modelling study. Lancet Oncol. 2020;21(8):1023-34.

$6 \mathrm{Wu}$ Z, McGoogan JM. Characteristics of and important lessons from the coronavirus disease 2019 (COVID-19) outbreak in China: summary of a report of 72,314 cases from the Chinese center for disease control and prevention. JAMA. 2020;323(13):1239-42.

7 Onder G, Rezza G, Brusaferro S. Case-fatality rate and characteristics of patients dying in relation to COVID-19 in Italy. JAMA. 2020; 323(18):1775-6.

8 Liang W, Guan W, Chen R, Wang W, Li J, Xu $\mathrm{K}$, et al. Cancer patients in SARS-CoV-2 infection: a nationwide analysis in China. Lancet Oncol. 2020;21(3):335-7.
9 Philouze P, Cortet M, Quattrone D, Céruse P, Aubrun F, Dubernard G, et al. Surgical activity during the Covid-19 pandemic: results for 112 patients in a French tertiary care center, a quality improvement study. Int J Surg. 2020; 80:194-201.

10 Lee LY, Cazier JB, Angelis V, Arnold R, Bisht V, Campton NA, et al. COVID-19 mortality in patients with cancer on chemotherapy or other anticancer treatments: a prospective cohort study. Lancet. 2020;395(10241):191926.

11 Peine A, Paffenholz P, Martin L, Dohmen S, Marx G, Loosen SH, et al. Telemedicine in Germany during the COVID-19 pandemic: multi-professional national survey. J Med Internet Res. 2020;22(8):e19745.

12 ESGE, ESGE and ESGENA Position Statement on gastrointestinal endoscopy and the COVID-19 pandemic. 2020. Available from: https: //www.esge.com/assets/downloads/ pdfs/general/ESGE_ESGENA_Position_ Statement_gastrointestinal_endoscopy_COVID_19_pandemic.pdf Accessed 2020 Aug 2.

13 American College of Gastroenterology. 2020. Available from: https://gi.org/2020/03/15/ joint-gi-society-message-on-covid-19/ Accessed 2020 Aug 2.

14 Blay JY, Soibinet P, Penel N, Bompas E, Duffaud F, Stoeckle E, et al. Improved survival using specialized multidisciplinary board in sarcoma patients. Ann Oncol. 2017;28(11): 2852-9.
15 Kehl KL, Landrum MB, Kahn KL, Gray SW, Chen AB, Keating NL. Tumor board participation among physicians caring for patients with lung or colorectal cancer. J Oncol Pract. 2015;11(3):e267-78.

16 CovidSurg C, Nepogodiev D, Bhangu A. Elective surgery cancellations due to the $\mathrm{CO}$ VID-19 pandemic: global predictive modelling to inform surgical recovery plans. $\mathrm{Br} \mathrm{J}$ Surg. 2020;107(11):1440-9.

17 Balakrishnan A, Lesurtel M, Siriwardena AK, Heinrich S, Serrablo A, Besselink MGH, et al. Delivery of hepato-pancreato-biliary surgery during the COVID-19 pandemic: an European-African Hepato-Pancreato-Biliary Association (E-AHPBA) cross-sectional survey. HPB. 2020;22(8):1128-34.

18 Tzeng CD, Teshome M, Katz MHG, Weinberg JS, Lai SY, Antonoff MB, et al. Cancer surgery scheduling during and after the COVID-19 first wave: the MD anderson cancer center experience. Ann Surg. 2020;272(2): e106-11.

19 Al-Omar K, Bakkar S, Khasawneh L, Donatini G, Miccoli P. Resuming elective surgery in the time of COVID-19: a safe and comprehensive strategy. Updates Surg. 2020;72(2):291-5.

20 NHS. 2020. Available from: https://www.england.nhs.uk/coronavirus/wp-content/uploads/sites/52/2020/04/second-phase-ofnhs-response-to-covid-19-letter-to-chief-execs-29-april-2020.pdf Accessed 2020 Aug 2.

21 Curigliano G. How to guarantee the best of care to patients with cancer during the COVID-19 epidemic: the Italian experience. Oncologist. 2020;25(6):463-7. 